\title{
Intelligent MAC and Routing Protocols for Wireless Sensor Networks
}

\author{
Murat Dener
}

\begin{abstract}
Energy consumption is one of the major limitations affecting design of Sensor Network. Efficient use of energy by sensor nodes is vital to lifetime of a Sensor Network. Studies on Sensor Networks, which still remain current in academia from its first use to present day, are increasing day by day. However, such studies are often limited to solving a specific problem, and no standards have been issued yet. On the other hand, several methods and techniques can produce positive effect in certain states of the Network as well as negative effects in other states. Considering these factors, Sensor Network should be self-optimized, real-time detect any changes and maintain its optimum state, and sensor nodes should operate in harmony and with minimum workload. A Sensor Network operating in this way will have a longer life cycle and use less power with maximum energy efficiency. This can be achieved by giving sensor nodes as well as Sensor Network comprised of these nodes smart approaches. In this context, this study suggests smart approaches to $\mathrm{MAC}$ and routing protocols.
\end{abstract}

Index Terms - Intelligent systems, MAC protocol, routing protocol, sensor network.

\section{INTRODUCTION}

Since power consumption by sensor nodes affects the life cycle of a Sensor Network, efficient use of power by these nodes is critical to a Sensor Network. Sensor Networks should therefore be designed to efficiently use available power, reduce power consumption by the nodes, and balance the power use in the network. The aim is to optimize life cycle of a Sensor Network and minimize the total consumption of power. Various protocols and algorithms have been developed for sensors to optimally use the energy and optimize the lifetime of the network. However, such solutions have been limited to solving a specific problem. This is because Sensor Networks vary depending on the application. In the circumstances, an implemented method, algorithm or protocol would yield favorable results for any Sensor Network but adverse results for another Sensor Network or different conditions of the same Sensor Network.

It is both costly and impossible to individually set behavior of a sensor in a Network with hundreds of sensor nodes. Sensor battery may run out, fail, or communication may be interrupted by an obstacle between after installing the network. Power consumption by Sensor Networks, energy efficiency, self-adaptation of a Sensor Network to any conditions, and optimum operation can only be accomplished by giving sensor nodes human-like behaviors with artificial

Manuscript received February 5, 2016; revised May 25, 2016.

Murat Dener is with the Graduate School of Natural and Applied Sciences, Gazi University, Ankara, Turkey (e-mail muratdener@gazi.edu.tr). intelligence techniques.

The future objectives of the study include installing protocols and algorithms to be developed in sensor nodes with artificial intelligence techniques; yielding practical outcomes by installing sensor network; giving sensor nodes human-like behaviors; how sensor nodes need to behave depending on the situation; and incorporating any solutions to any problems with power consumption-efficiency in the Sensor Network rather than dealing with a specific problem. Given that it is excessively costly and often impossible to replace or recharge the sensors and energy sources used in the operating environment, the extensive solution to be developed is expected to increase the life cycle of Sensor Network by 2 times with the same hardware.

This study includes what causes power consumption in MAC and Routing Protocols and suggests artificial intelligence techniques necessary for energy efficiency of these protocols.

\section{ARTIFICIAL INTELLIGENCE}

Intelligence can be defined as thinking, reasoning, perceiving objective facts, comprehending, judging, inference, abstraction, learning abilities and adaptation to new situations. Artificial intelligence is on the other hand intelligence of inorganic systems that have such characteristics above. In addition, Artificial Intelligence is also defined as ability of a computer or machine controlled by a computer to perform tasks of high mental processes such as reasoning, inferring, generalizing and learning from past experiences which are often thought to be human-specific qualities.

Artificial Intelligence is a multidisciplinary computer science that is fed on many different domains. These domains include Philosophy, Mathematics, Algorithm, Economics, Psychology, Computer Engineering, Neurology, Control Theory, Cybernetics, and Linguistics. The concept of artificial intelligence is as old as modern computer science. The originator is considered Alan M. Turing who raised the question "Can machines think?" The followings include the artificial intelligence techniques that could be used in sensor networks.

General characteristics and advantages of Artificial Intelligence technique are listed below.

1) Artificial intelligence learns the incidents and makes similar decisions for similar incidents.

2) It has information processing method different from known classical programming methods.

3) Its data processing system is installed on a network.

4) It uses samples of artificial intelligence techniques. It has 
an ability to learn adaptively.

5) Training set to train artificial neural networks and testing sets to measure performance of the network are installed.

6) It is able to produce information on unseen samples by generalizing the old samples.

7) It can be used for detection.

8) It is able to associate and classify the data.

9) It can complete the missing data.

10) It has an ability to adapt to new situations as it constantly receives new data input.

11) It can operate on missing data which is different from conventional systems.

12) It has fault tolerance since it can operate on missing data.

13) It is able to process incomplete ambiguous data.

14) No sudden failures occur, only failures at network level as it is tolerated against missing data and failure.

15) It has a distributed memory as data is processed by distributing to a network.

16) It can only operate on numeric data.

In addition to these features, each developed artificial intelligent model may have its own features.

\section{ANALYZE}

Situations causing power consumption and things to do for energy efficiency fall into two sections. These sections analyze the Sensor Networks in detail for power consumption/efficiency. One of the most significant limitations is power consumption when designing a Wireless Sensor Network. Power consumption of sensor nodes affect the life cycle of a Wireless Sensor Network. Therefore, efficient use of power by nodes is vital to Wireless Sensor Networks. There are many studies performed in academic and industrial fields to make power consumption efficient. These studies generally focus on Routing and MAC protocols [1].

\section{A. MAC Protocol}

Media Access Control (MAC) is a mechanism for effectively distributing wireless transmission medium between the nodes. MAC layer involves segmenting data packages, error recovery, transaction management, power protection and encryption. Operations on MAC layer in Sensor Networks are focused on reducing energy and delays. For the time being, it is not possible to create a standard MAC protocol on Sensor Networks but there are many studies on this topic in the literature [2].

The roles of MAC layer is listed below [3].

- Divides the data into "frames"; inserts address information (unit to send data) and error detection bits in this frame; controls incoming packets, address information, and error detection bits;

- Assesses the use of medium with respect to applications and performance index;

MAC protocols recommended for Wireless Sensor Networks fall into two groups, center and contention based. Center-based MAC protocols use features of TDMA method whereas contention-based MAC protocols are often based on CSMA/CA method [4]. In TDMA method, transmission time is divided in short equal time intervals. Each node transmits in a time interval specifically allocated to that node. Continuous communication is not possible because each node transmits in its time interval. Time intervals not used within transmission time remain empty. It saves energy for traffics where data transmission is not continuous. Attention should be paid when synchronizing as the nodes use the same band width. CSMA/CA method is based on "listen before speak" logic. Carrier Sense (CS) is utilized to identify usage of transmission medium, and Back-off algorithm is used to solve collision in the medium. Collision Avoidance is used for sensor nodes requesting transmission to access transmission medium in different time intervals. Access of sensor nodes to medium in different time intervals means access in a random time interval (Backoff time. Backoff time is a random layer of the time between the frames.

Both of the access methods can be used in the Network. In some cases, TDMA method becomes advantageous for energy and in other cases CSMA/CA method is advantageous. Dynamically enabling this equilibrium in accordance with changing conditions is possible with artificial intelligence techniques [5]. Sensor nodes are usually powered by a battery, and it is actually difficult and even impossible for a Sensor Network to replace or recharge the battery. Therefore, it is critical for the nodes to use the energy efficiently, which is already limited. In regard to a sensor node, communication unit has the largest share in energy consumption. Minimizing this will enhance the lifetime of the system. The causes of necessary energy consumption in MAC layer are listed below [6]-[9]:

1) Collision: Packets become unavailable when two packets collide being transmitted in overlapping time interval, thus the packets need to be resent. Resending means addition energy used. Collision also increases the delay.

2) Overhearing: Occurs when node unit receives a packet that has not been transmitted to it.

3) Control Packets: Power consumption will be adversely affected if there are too many control packets in the design. The large protocol headings (MAC, Network, etc.) of small data packets will also increase the power consumption.

4) Idle Listening: Receiver of radio chips must be always on for a node unit to receive the packets. This results in considerable power consumption despite no communication.

5) Traffic Fluctuation: The traffic has ups and downs in Transportation Data and Analysis (TDA). This, which is a matter of stability, adversely affects the power consumption.

In this sense, an energy-efficient MAC protocol that can be used in any sensor networks is achieved by resolving issues causing power consumption in MAC layer, designing access methods with artificial intelligence techniques.

\section{B. Routing Protocol}

Factors to consider for energy efficiency of routing in sensor networks are listed below:

In sensor networks, the basic power consumption is caused by transmission of node's own data and packets routed by nodes. Studies show that transmission of 1 bit of data from point $\mathrm{A}$ to point $\mathrm{B}$ results in power consumption equal to executing 1000 micro codes. Thus, minimizing the 
transmission, by performing as many as operations possible, can provide some improvement in power consumption. For example, compressing the data before sending and transmitting several packets in combination will reduce power consumption. Excessively increased processing load may result in power use much more than normal due to processing load. An appropriate balance needs to be established between these two factors [10]. Dynamically establishing such balance in accordance with changing conditions can be achieved by artificial intelligence techniques.

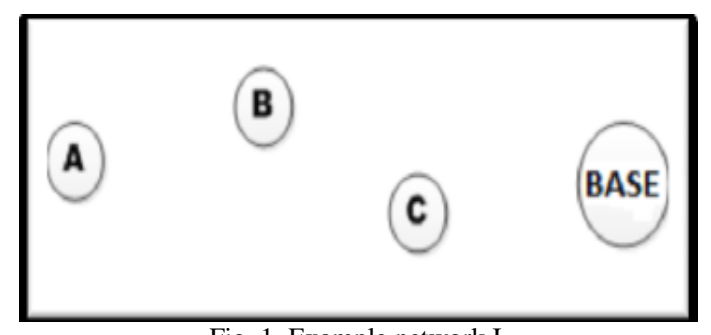

Fig. 1. Example network I.

As sensor networks are always routed to BASE station, higher power for routing is used by nodes close to the BASE station. As illustrated in Fig. 1, node C, the nearest node to the BASE station, is required to transmit both its own packet and packets of nodes A and B. Assuming that nodes A, B and C transmit packets in equal intervals, the power of node $C$ would run out at least 3 times before power of node A. Furthermore, depleted power of node $\mathrm{C}$ would break communication of nodes A and B with the BASE station. In this case, the nodes $A$ and B need to dynamically slow down, or improvements such as packet assembly should be considered, and the topology must be sustained throughout targeted operating time [11]. Detection of this and interventional methods can be provided by artificial intelligence techniques.

A node in a Sensor Network may have more than one neighbor. However, increased number of neighbors enhances the possibility of collision of transmitted packets in wireless networks where common communication pathway is used. Increased possibility of collision leads to packet repetition and unnecessary power use. Although solutions are sought using modulation techniques in the second layer, there is still something that can be done in the routing layer. Observation of number of collisions and errors will help determine who to speak or keep silent and when to speak or keep silent to prevent losses of packets [12]. However, in case of increased losses of packets, it can be dynamically set up that some nodes do not use some pathways.

Sensor nodes only rest or sleep as hardware when they are not active in transmitting. The nodes almost use no power during sleep and use power as much as in transmitting during resting. The measurements suggest that amount of power used by a sensor network during resting can be up to $70 \%$ of total power. This is a major problem to sensor networks. The reason why the nodes always rest is that they do not estimate when to receive a packet. In addition, many unnecessary packets may be received during resting. On the other hand, if everyone sleeps then a node requesting to transmit a packet may not find a respondent to route that packet. Ability of routing algorithm to determine who and when to sleep and who and when to wake up and rest will result in a large amount of energy save in topology. An effective solution needs to enable synchronization [13]. Again, all these can be achieved by artificial intelligence techniques.

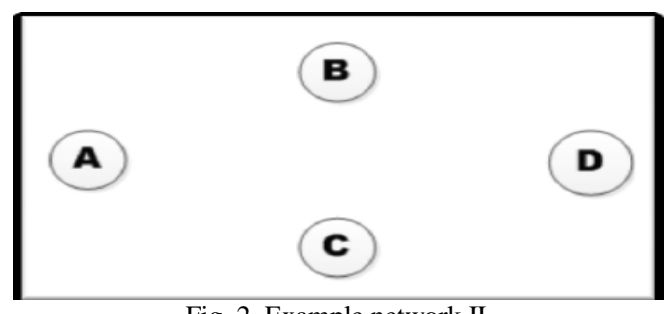

Fig. 2. Example network II.

Errors during packet transmission cause packet repetition. The rate of errors may be high in sensor networks. As depicted in Fig. 2, assuming that the error rate of connection between the node $\mathrm{C}$ and the node $\mathrm{D}$ is very high, routing packets sent from the node $\mathrm{A}$ via node $\mathrm{C}$ would result in unnecessary repetition of many packets. In fact, the packets can be sent via B with less error thus using less power [14]. Topologic power consumption will reduce if such connection with higher error rate is detected by routing algorithm and not used as much as possible. Weaker nodes than other nodes in terms of energy may be produced in sensor networks over time. Overall lifetime will increase if such nodes are identified and not used for routing. Fig. 3 illustrates an example of topology.

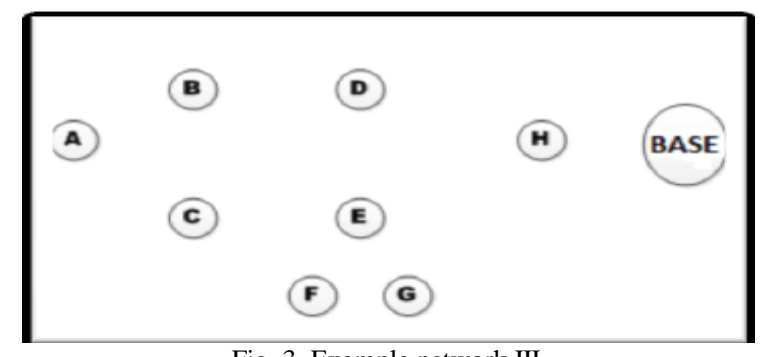

Fig. 3. Example network III.

Let us assume that node $\mathrm{E}$ has less energy than the other nodes. In this case, transmission of packets of nodes A and C through alternative paths will extend survival time of node $\mathrm{E}$. Furthermore, since the connection of nodes $F$ and $G$ to the BASE station is through node $\mathrm{E}$, lifetime of their connection will be extended [15]. There are many alternative paths from a node to the BASE station in sensor networks. Fair and balanced use of alternative paths, as much as possible, from the source node to the BASE station will extend the overall lifetime [16]. For example, if there are two alternative paths from a node A to the BASE station, detection and use of these two paths by routing algorithm will extend the overall lifetime of the network. The factors to consider for routing in sensor networks are listed below [17]-[20].

Unplanned placement: Sensor points are usually distributed randomly. System should be able to establish connections and make itself operable through this distribution. Connections should be able to rearrange due to errors of sensor points, and the system should be adaptive to changes in the network.

Adjustability of capacity use: There may be hundreds, thousands of, or more sensor points positioned in the sensing area. A diagram is needed to deal with such big numbers. In 
addition, changes in the size of network, number of points, or topology must not affect function of sensor points. However, routing protocols of a sensor network should be adjustable for adequate response to events in the environment. Most of the sensor nodes are in sleep mode until an event occurs. The remaining few sensor nodes detect roughly. After detection of occurrence of the event, system should be able to configure itself for more quality detection. Receiving data is more important than knowing private identity of source sender. The best solution to minimize the number of transmitted messages on the network is routing protocols because a very large amount of energy is used when transmitting data packets. The aim of many protocols is to minimize the power and maximize the lifetime of sensor networks because the nodes limits the energy, and capacity of memory and time for CPU processing. In this way, an energy-efficient routing protocol to be used by any sensor networks can be developed by eliminating what causes power consumption in the routing layer, and designing the factors to consider in routing with artificial intelligence techniques.

\section{CONCLUSION}

This study suggests redesigning MAC and routing protocols using artificial intelligence techniques in order to eliminate anything that results in power consumption in sensor networks and improve energy efficiency. In the next study, sensor nodes will be taught any possible situations from initial time of the network to the end, and these nodes will be equipped with human-like behaviors. For this, high cognitive functions specific to human mind such as sense, learning, attribution of multiple concepts, thinking, reasoning, solving problems, communicating, deducing, and making a decision, or Artificial Intelligence techniques, which is an artificial operating system expected to display autonomous behaviors, (e.g. optimization algorithms, fuzzy logic, genetic algorithms, expert systems, artificial neural networks, local search, hybrid intelligent systems, dynamic programming, split and cut, simulated annealing, taboo search, hill climbing, ant colony, particle swarm optimization) will be used. Firstly, the problems with power consumption-efficiency will be extensively analyzed since the study will be implemented practically. Later, what technique should be used will be decided by criteria for power-memory-operating capacity to deal with each problem. More than one method will be used during decision-making process to select the best one from the criteria. GSM/GPRS+GPS Modules will be integrated in sensor nodes representing cluster heads and base stations. In this way, changes in power-delay-performance in a Sensor Network and data received by cluster heads or base station can be monitored on a mobile / web basis. In case of implementation of the future study, it will surely make a substantial contribution to applications in the Sensor Networks in terms of energy efficiency.

\section{REFERENCES}

[1] I. F. Akyildiz, W. Su, Y. Sankarasubramaniam, and E. Cayirci, "A survey on sensor networks," IEEE Commun. Mag., vol. 40, no. 8, pp. $102-114,2002$
[2] I. Demirkol, C. Ersoy, and F. Alagöz, "MAC protocols for wireless sensor networks: A survey," IEEE Communications Magazine, vol. 44, no. 4, pp. 115-121, 2006.

[3] T. Van Dam and K. Langendoen, "An adaptive energy-efficient MAC protocol for wireless sensor networks," in Proc. the First ACM Conference on Embedded Networked Sensor Systems, Los Angeles, CA, USA, 2003, pp. 171-180.

[4] A. El-Hoiydi, "Spatial TDMA and CSMA with preamble sampling for low power Ad Hoc wireless sensor networks," in Proc. ISCC 2002, 2002, pp. 685-692.

[5] M. Dener and Ö. F. Bay, "Medium access control protocols for wireless sensor networks: Literature survey," G.U. Journal of Science, vol. 25 no. 2, pp. 455-564, 2012.

[6] W. Ye, J. Heidemann, and D. Estrin, "An energy-efficient MAC protocol for wireless sensor networks," in Proc. Twenty-First Annual Joint Conference of the IEEE Computer and Communications Societies (INFOCOM), 2002, vol. 3, pp. 1567-1576.

[7] P. Lin, C. Qiao, and X. Wang, "Medium access control with a dynamic duty cycle for sensor networks," IEEE WCNC, vol. 3, pp. 1534-1539, 2004.

[8] W. Ye, J. Heidemann, and D. Estrin, "Medium access control with coordinated adaptive sleeping for wireless sensor networks," IEEE/ACM Trans. Net., vol. 12, no. 3, pp. 493-506, 2004.

[9] C. Ceken, "An energy efficient and delay sensitive centralized MAC protocol for wireless sensor networks," ELSEVIER Science Direct Computer Standards \& Interfaces, 2007.

[10] A. P. Nikolaos, A. N. Stefanos, and D. V. Dimitrios, "Energy-efficient routing protocols in wireless sensor networks: A survey," IEEE Communications Survey \& Tutorials, vol. 15, no. 2, 2013.

[11] S. Ehsan and B. Hamdaoui, "A survey on energy-efficient routing techniques with QoS assurances for wireless multimedia sensor networks," IEEE Commun. Surveys Tuts., vol. 14, issue 2, pp. 265-278, 2011.

[12] R. V. Biradar, V. C. Patil, S. R. Sawant, and R. R. Mudholkar, "Classifiacation and comparison of routing protocols in wireless sensor networks," Special Issue on Ubiquitous Computing Security Systems, vol. 4, issue 2, pp. 704-711, 2009.

[13] L. J. G. Villalba, L. S. Orozco, A. T. Cabrera, and C. J. B. Abbas, "Routing protocols in wireless sensor networks," Sensors, vol. 9, pp. 8399-8421, 2009.

[14] E. Gelenbe and C. H. Ngai, "Adaptive QoS routing for significant events in wireless sensor networks," in Proc. the 5th IEEE International Conference on Mobile Ad Hoc and Sensor Systems, 2008, pp. 410-415.

[15] K. Akkaya and M. Younis, "A survey on routing protocols for wireless sensor networks," Elsevier Ad Hoc Networks, vol. 3, no. 3, 2005.

[16] K. Akkaya and M. Younis, "An energy-aware QoS routing protocol for wireless sensor networks," in Proc. the IEEE Workshop on Mobile and Wireless Networks, Providence, Rhode Island, 2003.

[17] J. N. Al-Karak and A. E. Kamal, "Routing techniques in wireless sensor networks: A survey," IEEE Wireless Communications, vol. 11, 2004.

[18] J. H. Chang and L. Tassiulas, "Maximum lifetime routing in wireless sensor networks," Transactions on Networking, vol. 12, no. 4, 2004.

[19] C. Rahul and J. Rabaey, "Energy aware routing for low energy ad hoc sensor networks," in Proc. IEEE Wireless Communications and Networking Conference (WCNC), 2002, Orlando, USA, vol. 1, pp. 350-355.

[20] C. Schurgers and M. B. Srivastava, "Energy efficient routing in wireless sensor networks," in Proc. on Communications for Network-Centric Operations: Creating the Information Force, McLean, VA, 2001.

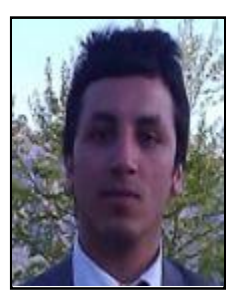

Murat Dener received his M.Sc. and Ph.D. degrees in electronic and computer education from Gazi University, Turkey, in 2008 and 2012, respectively. His doctorate thesis entitled by Design and Implementation of a Secure Data Link Layer Protocol for Wireless Sensor Networks. He worked in Georgia Tech E-Stadium Team in 2011. From 2005 to 2012, he was a research assistant in the Graduate School of Natural and Applied Sciences. Since 2012, he is a doctor in Gazi University. His research interest includes the next-generation wireless networks, wireless Ad Hoc and sensor networks, cognitive radio networks. 